\title{
Multimodal Authentication using Tensor Algebra
}

\author{
K. Pavan Kumar ${ }^{1}$, PESN Krishna Prasad ${ }^{2}$, Y Suresh ${ }^{3}$, P. Sitarama Murthy ${ }^{4}$ \\ ${ }^{1}$ Asst.Prof, Dept. of IT ,PVP Siddhartha Institute of Technology, India, pavankalluri@pvpsiddhartha.ac.in \\ ${ }^{2}$ Professor, Dept of CSE, Sri Venkateswara College of Engineering, India, surya125@ gmail.com \\ ${ }^{3}$ Asst.Prof, Dept. of IT,PVP Siddhartha Institute of Technology, India, suresh@pvpsiddhrtha.ac.in \\ ${ }^{4}$ Assoc. Prof, Dept of IT, Vishnu Institute of Technology, India, psramam.1@ gmail.com
}

\begin{abstract}
S
Tensors play a major role in the process of image patterns by considering the concept of approximations by varying orders of polynomials. Tensors define a normal decomposition of uniform patterns that leads the connection between the symmetric tensors and multivariate polynomials. One of the inductive learning concepts is feature selection. The selection of a subset of features from the given list of features is by considering an evaluation measure i.e., the subset of features with specified size, the smaller size subset features satisfies with certain restriction on the chosen measure and the set with best features among its size and the value of its measure. The objective of this is to improve the inductive learning process. Feature extraction and selection is one key concept for the selection of best features in order to analyze and represent the patterns through the concept of Tensor objects. Numerous traditional approaches exist for the extraction of features in the process of image patterns such as PCA, Sparse PCA, Kernel PCA, SVD, and Sparse SVD. Among these approaches, to analyze the tensor features for the classification and recognition tensor based matrix factorization techniques for the extraction and selection of features. In this, by considering biometrics features such as face and finger of a person in multimodal authentication system by applying Nonnegative Tucker Factorization (NTF) for feature selection and extraction, Principle Component Analysis (PCA) for image fusion. Finally, results were proposed.
\end{abstract}

Key words: Tensors, PCA, NTF, SVD.

\section{INTRODUCTION}

Throughout the world wide the statistical study of biometric characteristics such as palm, iris, vein and face are have been accepted and using in different areas such as educational organizations, air port authorities, government agencies and to implement various Government beneficiary schemes and also in other institutions to authenticate the person. Each trait has its own features. But, successful implementation of biometric systems in these areas does not imply ideal security system and also proper identification is a crucial part of admission control that makes important to any security system. Identification of person generally based on the user knows such as PIN or password and user has smart card.

Biometric[1] is systematic process that mostly focus on methods of verification and or validation depends on the behavioral or physiological individuality. These characteristics are not duplicate and convenient. Most of the biometric systems are unimodal i.e., the person can be authorized based on the probabilistic conditions, are not up to the mark due to lack of noisy sensor data and individuality. To regulate these problems in unimodal system, researchers are implementing multimodal biometric systems [6,7], focuses on two or more traits as mixture either in blend based or non blend based from the same person in order to authenticate and validation. This kind of systems provides more accuracy than the unimodal systems and also availability of the software and computing devices.

As Specified in [2], any biometric system used for authentication is a layer by layer process, which consists of acquisition, feature extraction by eliminating the noise, and finally maintain in the repositories. After enrolment, identification step takes place and focuses on the acquisition, feature Extraction, comparison, and decision steps. In these methods, Feature extraction plays a key role in all the mentioned phases. The extracted features can be fusioned by using mathematical methods such as PCA, LDA and its variants. In the next step, the features can be obtained using statistical techniques such as LU, SVD to produce key components. Also we can apply Kronecker components $[3,4,5]$ to calculate the weights to make the system very complex to reduce the error rate. Finally in the decision approach different error rate procedures will be applied. Based upon the chose threshold value the acceptance or rejection depends. Due to advantages of vector logic, it is implemented in the process of fusioning the patterns, extraction of features, generating keys and computation for matching process. To improve the learning process, tensor algebra is also applied. 
K. Pavan Kumar et al., International Journal of Emerging Trends in Engineering Research, 8(9), September 2020,5091 - 5094

\subsection{Vector Logic}

In the areas of computer vision and image processing vector logic is the most driving force due to representation of image in the form of a matrix. On these matrices representations different types of computations procedures are applied such as calculation of eigen values and eigen vectors and also used to represent feature vectors. In matrix algebra, Kronecker product is one of the key product[8], which computes in the areas of image segmentation, analysis and security models. The data elements can be represented as monadic, dyadic and so on. In monadic, the data is represented as one dimensional, where as in dyadic representation data is stored in the form of rows and columns [9].

\subsection{Tensor Algebra}

A pixel is a scalar quantity whose specification requires just one number. On the other hand, with respect to some basis, vector measurement requires three numbers namely its components. For a general object tensor, vectors and scalars are special cases of order $\mathrm{n}$, whose order in any coordinate system requires 3 n numbers, called the components of tensor. In fact, scalars and tensors are order of zero, one with $3^{\circ}=1$ and $3^{1}=3$ components [9].

\subsection{Tensor Objects:}

The decomposition of a multi-linear tensor $\mathrm{T}$ of an order 3 is given in the below figure:
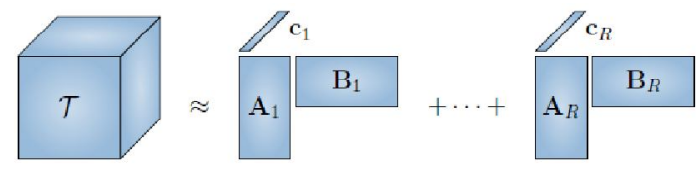

Figure 1: Visual Representation of Multi-Linear Tensor of an order 3 .

\subsection{Tensor Representation}

In physics, "Tensor" generally refers to a tensor field, which is a generalization of a vector field. With position it varies continuously with each point of a geometric space. Using multidimensional arrays multi-dimensional data can be represented, referred as tensors in mathematics. The number of dimensions $N$ defines the order of a tensor, and the elements (entries) of a tensor are addressed by $N$ indices.

The different types of Tensor representations are as follows:

\section{a. Direct sum representations}

Let $(\rho \mathrm{V}, \mathrm{V})$ and $(\rho \mathrm{W}, \mathrm{W})$ be representations of a finite group $\mathrm{G}$ over complex vector spaces $\mathrm{V}$ and $\mathrm{W}$ of dimensions $\mathrm{n}$ and $\mathrm{m}$, respectively [8]. We can create a new vector space $\mathrm{V} \oplus \mathrm{W}$ by taking the direct sum of $\mathrm{V}$ and $\mathrm{W}$ :

$$
\mathrm{V} \oplus \mathrm{W}:=\{(\mathrm{v}, \mathrm{w}) \mid \mathrm{v} \in \mathrm{V}, \mathrm{w} \in \mathrm{W}\}
$$

If $\{\mathrm{e} 1, \ldots, \mathrm{en}\}$ and $\{\mathrm{f} 1, \ldots, \mathrm{fn}\}$ are respective bases for $\mathrm{V}$ and $\mathrm{W}$, then

$$
\{(\mathrm{e} 1,0), \ldots,(\mathrm{en}, 0),(0, \mathrm{f} 1), \ldots,(0, \mathrm{fm})\}
$$

is a basis for $\mathrm{V} \oplus \mathrm{W}$, hence it is $\mathrm{n}+\mathrm{m}$-dimensional. Given $\rho \mathrm{V}$ and $\rho \mathrm{W}$ above, we have a natural representation $\rho \mathrm{V} \oplus \mathrm{W}$ of $\mathrm{G}$ on $\mathrm{V} \oplus \mathrm{W}$ by letting

$$
\rho \mathrm{V} \oplus \mathrm{W}(\mathrm{g})(\mathrm{v}, \mathrm{w}):=(\rho \mathrm{V}(\mathrm{g}) \mathrm{v}, \rho \mathrm{W}(\mathrm{g}) \mathrm{w}) .
$$

To put it in terms of matrices, we have that $\rho \mathrm{V} \oplus \mathrm{W}$ $(\mathrm{g})$ is an $(\mathrm{n}+\mathrm{m}) \times(\mathrm{n}+\mathrm{m})$ block diagonal matrix of the form

$$
\left[\begin{array}{cc}
\rho V(g) & 0 \\
0 & \rho W(g)
\end{array}\right]
$$

where $\rho \mathrm{V}(\mathrm{g})$ and $\rho \mathrm{W}(\mathrm{g})$ are matrices of dimensions $\mathrm{n} \times \mathrm{n}$ and $\mathrm{m} \times \mathrm{m}$, respectively.

\section{b. Tensor product representations}

Again using $(\rho \mathrm{V}, \mathrm{V})$ and $(\rho \mathrm{W}, \mathrm{W})$, we can create yet another vector space $\mathrm{V} \otimes \mathrm{W}$, called the tensor product of $\mathrm{V}$ and $\mathrm{W}$. Formally, we define

$$
\begin{aligned}
& \mathrm{V} \otimes \mathrm{W}:=\mathrm{n} \text { finite sums } \mathrm{X} \lambda \mathrm{ij}(\mathrm{vi} \otimes \mathrm{wj}) \mid \lambda \mathrm{ij} \in \mathrm{C} \text {, vi } \\
& \in \mathrm{V}, \mathrm{wj} \in \mathrm{W} \text { o }
\end{aligned}
$$

where the terms $\lambda(\mathrm{v} \otimes \mathrm{w})$ are bilinear in $\mathrm{C}$. That is, for any $\lambda \in \mathrm{C}, \mathrm{v} \in \mathrm{V}, \mathrm{w} \in \mathrm{W}$, we have

$$
\begin{array}{cl}
\text { i. } & \lambda(\mathrm{v} \otimes \mathrm{w})=\lambda \mathrm{v} \otimes \mathrm{w}=\mathrm{v} \otimes \lambda \mathrm{w} \\
\text { ii. } & (\mathrm{v} 1+\mathrm{v} 2) \otimes \mathrm{w}=(\mathrm{v} 1 \otimes \mathrm{w})+(\mathrm{v} 2 \otimes \mathrm{w}) \\
\text { iii. } & \mathrm{v} \otimes(\mathrm{w} 1+\mathrm{w} 2)=(\mathrm{v} \otimes \mathrm{w} 1)+(\mathrm{v} \otimes \mathrm{w} 2) .
\end{array}
$$

\section{TENSOR BASED APPROXIMATION TECHNIQUES}

A traditional family of methods for extracting and selecting the features from image data is based on the decomposition techniques in the form of matrices formed by all input data as columns. The input data matrix with existing features is transformed into a new data matrix using the new features.

\section{A. Tensor based Feature Selection methods (TFS)}

From the observation of traditional feature extraction/selection method SVD decomposition $\mathrm{X}=\mathrm{UVW}$, the data(columns) of $\mathrm{U}$ are the eigen vectors of the matrix $\mathrm{XX}^{\mathrm{t}}$, whereas the data(rows) of $\mathrm{W}$ are the eigen vectors of the matrix $X^{t} X$. Eigen vectors are orthogonal and therefore $\mathrm{U}^{t} \mathrm{U}=\mathrm{I}$ and $\mathrm{WW}^{\mathrm{t}}=\mathrm{I}$. by applying the dimensionality reduction, the approximation $X=U n V n W n$ is minimizing the matrix representation error

$$
\epsilon=\|X-\hat{X}\|^{2}=\operatorname{trace}\left\{(X-\hat{X})\langle X-\hat{X}\rangle^{t}\right\}
$$


Generalized version of SVD modifies the orthogonal constraints such that $\mathrm{U}^{\mathrm{t}} \mathrm{C}_{\mathrm{u}} \mathrm{U}=\mathrm{I}$ and $\mathrm{WCwW}^{\mathrm{t}}=\mathrm{I}$, where $\mathrm{C}_{\mathrm{u}}$ and $\mathrm{C}_{\mathrm{w}}$ are the two constraints matrices. By performing the reduction process, the approximation is the one minimizing

$$
\left\{\in=\operatorname{trace}\left\{C u(X-\hat{X}) C w\langle X-\hat{X}\rangle^{t}\right\}\right.
$$

Thus, Generalized SVD is a very versatile tool by the consideration of constrains matrices that can be particularized to correspondence analysis.

B. Multi-Linear Singular Value Decomposition (MLSVD)[10] is one of the tensor based decomposition method for generalization of pair-wise symmetric tensors through symmetric eigen value decomposition strategy. The concept of MLSVD is an appropriate generalization of the link the row (column) vectors and the right (left) singular values of the matrix. A simple representation of MLSVD is depicted as in figure 4.2. Let $\mathrm{T}$ be the tensor of size $I_{1} X \quad I_{2} X \quad I_{3} \ldots . X \quad I_{N}$ as a multi-linear tensor matrix product of core tensor $\mathrm{S}$ with size $R_{1} X R_{2} X R_{3} \ldots \ldots$. $X R_{N}, U^{(n)}$ factors of $\mathrm{N}$, size $I_{n} X R_{n}$

$$
\approx S \cdot{ }_{1}^{1} U \cdot{ }_{2}^{2} U \cdot{ }_{3}^{3} U \ldots . .{ }_{N}^{N} U \text {. }
$$

Where $\tau$ is a tensor matrix product of mode $n$.

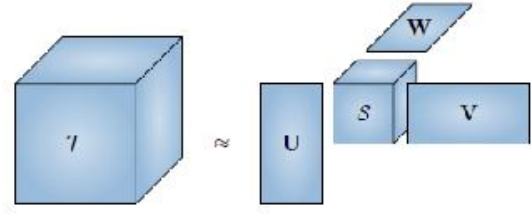

Figure 2: Multi-Linear Singular Value Decomposition

C. Multi-Linear Principal Component Analysis (MLPCA)[11]is one of the important PCA algorithm with Tensor based feature extraction methods to extract the features in the form tensor objects from the image patterns. It's a new way of dimensionality reduction and feature extraction method in which performing the tensor modes that allows to capture the projected tensors from the original tensor patterns to determine the low dimensionality. In the analysis of MLPCA, the projection refers to multi linear projections that transforms into independent subspace projections and then obtained eigenvectors with comprised patterns directly for later usage. Such eigenvectors can be considered as subspace tensor features often called tensor objects for better analysis with classification and prediction approaches. The detailed procedure for MLPCA is discussed in [11]

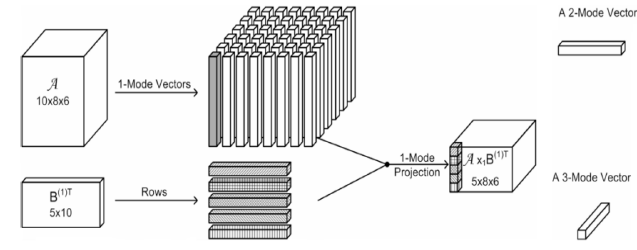

Figure 3: Detailed presentation of multi-linear projection using MLPCA
D. Nonnegative Tucker Factorization (NTF) is a recent multi-way extension of nonnegative matrix factorization (NMF) [13]. Tucker decomposition decomposes a tensor into set of matrices and one small core tensor. This is used as an experimental tool. For illustration, it is used to represent the threeway data by means of relatively small numbers of components for each of the three or more modes, and with three or higher dimensional array the components are linked to each other.

For the extraction of nonnegative parts-based and physically meaningful latent components from highdimensional tensor data while preserving the natural multi-linear structure of data Nonnegative Tucker Factorization (NTF)[13] is an important tool. But NTF algorithms from a very high computational complexity in terms of both storage and computation time as the data tensor often has multiple modes and is large-scale which has been one major obstacle for practical applications.

Principal Component Analysis (PCA): In principal component analysis, we find the directions in the data with variation, i.e., the eigen vectors resultant to the largest eigen values of the covariance matrix, and project the data onto these directions. i.e. we might discard important non second order information by PCA. If we denote the matrix of eigen vectors sorted according to eigen value by $\widetilde{U}$, then PCA transformation of the data as $\mathrm{Y}=\widetilde{U}^{\mathrm{T}} \mathrm{X}$. The eigen vectors are called as principle components [12, 14]. By selecting the first d rows of $\mathrm{Y}$, we have projected the data from $\mathrm{n}$ down to d dimensions.

\section{Decision Strategy}

In this paper, Mean Square Error (MSE) is considered as decision strategy for recognition of chosen patterns. The Mean Square Error (MSE) of an estimator $\widehat{X}$ of a parameter $\mathrm{X}$ is the function of $\mathrm{X}$ is defined by $\mathrm{E}(\widehat{\mathrm{X}}-\mathrm{X})^{2}$ and it is denoted as $\mathrm{MSE}_{\widehat{\mathrm{X}}}$ [14].

$$
\operatorname{MSE}(\widehat{X})=E\left[(\widehat{X}-X)^{2}\right]
$$

The Mean Square Error is equal to the sum of the variance and the squared bias of the estimator or of the predictions. In the case of the MSE of an estimator [9,14],

$$
\operatorname{MSE}(\widehat{X})=\operatorname{Var}(\widehat{X})+(\operatorname{Bias}(\widehat{X}, X))^{2}
$$

\section{EXPERIMENTAL RESULTS AND ANALYSIS}

In this paper, initially we applied pre processing for the training images. Next features can be extracted using Nonnegative Tucker Factorization (NTF). After getting the features apply the fusion by using the Principle Component Analysis (PCA). Once fusion can be done these features can be encoded using Kronecker Product and stored these results in memory. Similarly take the testing images and apply the pre-processing and features can be extracted using Nonnegative Tucker Factorization (NTF). After 
getting the features apply the fusion by using the Principle Component Analysis (PCA). Once fusion can be done these features can be encoded using Kronecker Product and stored these results in memory. Now these testing results can be compared with the training results by using distance metric Mean Square Error(MSE). Now threshold can be set and compare resultant value of MSE with this threshold. Based on that take the decision weather that testing image is known image or unknown image. The following diagram shows the flow of the implementation.

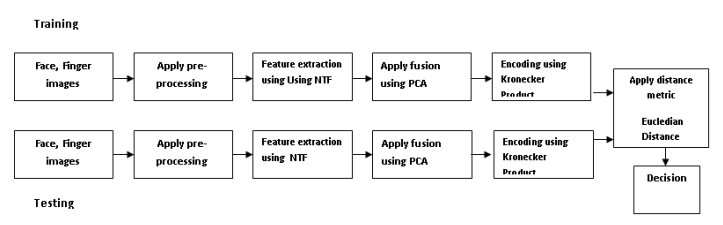

Figure 4: Data flow of the proposed model

Here threshold $d=2.5$ can be used. False acceptance rate and false rejection rates are verified on these data sets. The following table shows the results of NTF

Table 1: Results obtained by using NTF.

\begin{tabular}{|c|c|c|c|}
\hline \multirow{2}{*}{ S. No } & \multirow{2}{*}{ Key Size } & \multicolumn{2}{|c|}{ MSE } \\
\cline { 3 - 4 } & & Similar & Dissimilar \\
\hline 1 & $8 \times 8$ & 1.6384 & 3.6376 \\
\hline 2 & $16 \times 16$ & 1.4242 & 2.913 \\
\hline 3 & $24 \times 24$ & 1.7046 & 2.889 \\
\hline 4 & $32 \times 32$ & 1.7746 & 3.0296 \\
\hline 5 & $40 \times 40$ & 1.8964 & 3.0632 \\
\hline 6 & $48 \times 48$ & 2.0242 & 3.0488 \\
\hline 7 & $56 \times 56$ & 2.4564 & 3.222 \\
\hline 8 & $64 \times 64$ & 1.6384 & 3.6376 \\
\hline
\end{tabular}

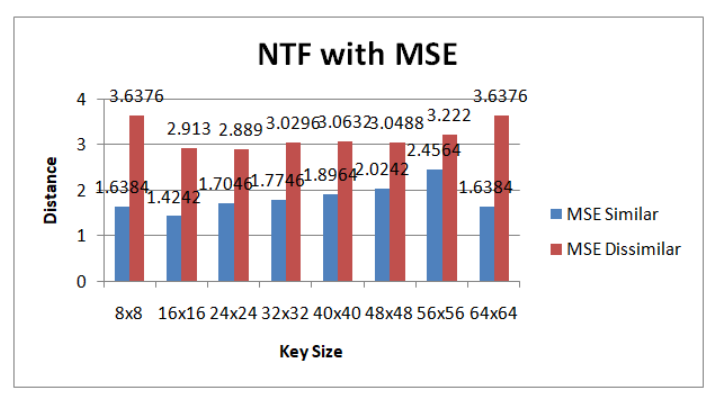

Figure 5: Results graph using NTF factorization

\section{REFERENCES}

1. K. Jain, A. Ross Prabhakar, S. An Introduction to Biometric Recognition. IEEE Trans. Circuits Syst. Video Technol., 14, 4-20, 2004.
2. Vaclav Matyas, ZdenekRiha, "Biometric Authentication -Security and Usability", http://www.fi.muni.cz/usr/matyas/

3. John W. Brewer, Kronecker Products and Matrix Calculus in System Analysis, IEEE Transactions on Circuits and Systems, Vol. 25, No. 9, 1978.

4. Lester Lipsky and Appie van deLiefvoort, Transformations of the Kronecker Product of Identical Servers to Reduced Product Space, 1995.

5. Wolfgang Hackbusch, Boris N. Khoromskij, Hierarchical Tensor-Product Approximations, 1-26.

6. V. Ghattis, A.G. Bors and I. Pitas, “ Multimodal decision level fusion for person authentication," IEEE Trans. Systems, Man and Cybernatics, vol. 29, no. 6, pp, 674-680, Nov. 1999.

7. https://stat.ethz.ch/R-manual/R-devel/library/ Matrix/html/KhatriRao.html

8. H. V. Henderson, F. Pukelsheim and S. R. Searle. On the history of the Kronecker product. Linear and Multilinear Algebra. 14:113-120, 1983.

9. Y. Suresh, K. Pavan Kumar, PESN Krishna Prasad, "Pattern Recognition Using Context Dependent Memory Model (CDMM) In Multimodal Authentication System", International Journal in Foundations of Computer Science \& Technology (IJFCST), Vol.5, No.1, PP.4757, January 2015.

10. Rajiv Kumar, M.Arthanari, M.Sivakumar, "Image Segmentation using DiscontinuityBased Approach", IJMIP, Volume 2, Issues 1/2, March/June 2012

11. Amari, S., Cichocki, A., Yang, H.H., A new learning algorithm for blind signal separation. Adv. Neural Inform. Process. Syst., 1996, 757-763.

12. S.Ribaric, I. Fratic and K. Kris, "A biometric verification system based on the fusion of palmprint and face features," Proc. Fourth Int. Symposium Image and Signal Processing, pp. 12-17, 2005.

13. http://www.comp.hkbu.edu.hk/v1/

14. Y Suresh -. "Image Analysis using LU, SVD and Kronecker Algebra in Multimodal Biometric Authentication System", International Journal of Research in Advent Technology, 2019 